\title{
Prevalence and Associated Factors of Regular Nonsteroidal Anti-inflammatory Drugs used in a Rural Community, Thailand
}

\author{
Pongsom Luanghirun ${ }^{1}$, Patid Tanaboriboon ${ }^{1}$, Pawaris Mahissarakul ${ }^{1}$, Chanikarn Tongruang ${ }^{1}$, Chanita \\ Chaichirawiwat ${ }^{1}$, Phunlerd Piyaraj ${ }^{2}$, Ploypun Narindrarangkura ${ }^{3}$ \& Nawachai Lertvivatpong ${ }^{4}$ \\ ${ }^{1} 5^{\text {th }}$ year Medical Cadet, Phramongkutklao College of Medicine, Thailand \\ ${ }^{2}$ Department of Parasitology, Phramongkutklao College of Medicine, Thailand \\ ${ }^{3}$ Department of Military and Community Medicine, Phramongkutklao College of Medicine, Thailand \\ ${ }^{4}$ Department of Pharmacology, Phramongkutklao College of Medicine, Thailand \\ Correspondence: Nawachai Lertvivatpong, Department of Pharmacology, Phramongkutklao College of Medicine, \\ 315 Ratchawithi Rd Ratchathewee BKK 10400, Thailand. Tel: 66-23-54-7752.
}

Received: May 28, 2017 Accepted: June 24, 2017 Online Published: June 30, 2017

doi:10.5539/gjhs.v9n9p58 URL: https://doi.org/10.5539/gjhs.v9n9p58

\begin{abstract}
Background: In Thailand, $67.2 \%$ of the population widely uses analgesics including nonsteroidal anti-inflammatory drugs (NSAIDs), which may lead to serious side effects. However, the information of regular NSAIDs used in Thailand is still limited.

Methods: A mixed method cross-sectional study was conducted. Quantitative data were collected using questionnaires to determine the prevalence and factors associated with regular NSAID use. The qualitative study was conducted using group and in-depth interviews to determine the knowledge, attitudes and practices of NSAID users.

Results: Of 771 participants, the prevalence of NSAID use was 31.1 and regular NSAID use was 7.4. Age, pain at the hips or thighs and pain score were independent factors associated with regular NSAID use. The qualitative study indicated that the use of NSAIDs was influenced by drug effectiveness, sources of NSAIDs and consideration of benefits and risks of the drugs.

Conclusion: This was the first report on the prevalence and associated factors of regular NSAID use in Thailand. In this community, nonprescribed NSAIDs might cause some serious side effects and undesirable drug interaction. Information on side effects of pain medications should be disseminated to the public including guidelines on how to use pain medications.
\end{abstract}

Keywords: Nonsteroidal anti-inflammatory drugs (NSAIDs), Prevalence, Associated risks, Rural community, Thailand, Primary data

\section{Introduction}

Nonsteroidal anti-inflammatory drugs (NSAIDs) are widely used in medical therapeutics (Somia \& Maria, 2014). Thus, they are prescribed to many patients, especially regarding musculoskeletal diseases. Data from the US found that people taking aspirin or NSAIDs regularly (taking aspirin or NSAIDs at least three times weekly more than three months in one year) comprised more than 43 million (19.0\%) and 29 million people (12.1\%), respectively and equal to prescribing NSAIDs more than 100 million times annually at a cost of more than 4.8 billion USD (Zhou et al., 2013). Regular NSAID use may cause some serious side effects especially gastrointestinal, cardiovascular and renal systems (Lertsrisatit \& Jantawiyanuchit, 2014; Polónia, 1997; Page \& Henry, 2000; Sorensen et al., 2000; Laine, 2004; Wolfe, Lichtenstein, \& Singh, 1999; Pope, Anderson, \& Felson, 1993; Winkelmayer, Waikar, Mogun, \& Solomon, 2008; Radford et al., 1996; Abraham \& Keane, 1984).

Data from the $4^{\text {th }}$ Thai National Health Survey Report by physical examination four times a year from 2008 to 2009 found that the population of Thailand is taking numerous painkillers (Akepalakorn, 2009). Considering the frequency of use found that people who took two to three days a week totaled $20.9 \%$ and those who used nearly every day and every day comprised 4.6 and $2.3 \%$, respectively. By age group, prevalence of pain medications increased with age and the highest among those aged 70 to 79 years. Populations living outside municipalities 
regularly consumed painkillers more than those living in municipalities. Populations in the northeast had the highest percentage $(2.8 \%)$, followed by the central region. Sources of analgesics constituted health centers or hospitals $(60.0 \%)$ and others from a pharmacy or retail store (Akepalakorn, 2009).

However, high prevalence of painkiller use was indicated, but the data of NSAID use in Thailand is limited. Thus, we have been interested in determining the prevalence and factors associated with regular NSAID use in a rural community. Thus, the cross-sectional study was conducted in a rural community, Chachoengsao Province, central Thailand. Both qualitative and quantitative methods were performed.

We gratefully acknowledge support, including funds and operation, from the Department of Military and Community Medicine, Department of Pharmacology, Phramongkutklao College of Medicine. We have no Conflict of interest to declare.

The researchers had interested in the prevalence of NSAIDs used in Baan Na Yao, Sanam Chai Khet District, Chachoengsao Province, and finding associated risk factors in the population to remind the situation and size of problems. The primary data will be made awareness of NSAIDs used in rural areas, clearly understanding characteristics of users, and enlightening associated factors using of the drugs. The data from this study can be reproduced and policies may be planned to reduce the using of NSAIDs and leading to reducing side effects that may occur from taking the drugs.

\section{Method}

A mixed method study was conducted in 2015 comprising a quantitative approach to determine prevalence and associated factors of NSAIDs using standardized questionnaire and a qualitative approach using group and in-depth interviews to describe the situations and problems of NSAIDs in the community. The study protocol was reviewed and approved by the Ethics Committee of the Royal Thai Army Medical Department. Informed consent was obtained from enrolled participants who completed a questionnaire and the providers of NSAIDs following standard protocols.

\subsection{Study Population}

The study was conducted in a rural community, Baan Na Yao Village, Sanamchaiket District, Chachengsao Province, central region of Thailand. Meanwhile in the central region, the people had immigrated from the northeastern and remained their traditional culture and lifestyle.

\subsection{Participant Characteristics}

Inclusion criteria

- People who had 18 years and older and lived in this area in November 2015

- People who had signed in the consent form

Exclusion criteria

- People who unable to communicate e.g. deftness, blindness or mute

Measures and Covariates

Primary Outcomes

1. Prevalence of regular NSAIDs used

2. Associated factors of regular NSAIDs used

Secondary Outcomes

1. Prevalence of NSAIDs Used

2. Types of NSAIDs

3. Sources of NSAIDs

\subsection{Quantitative Study}

\subsection{Sample Size Calculation}

Due to the previous study in Trends in the use of aspirin and nonsteroidal anti-inflammatory drugs in the general U.S. population (Zhou Y, 2013), the prevalence of regular NSAIDs used were 12\%. The sample size culculating with infinite population were

$$
n=\frac{Z^{2}(P)(1-P)}{d^{2}}
$$


$\mathrm{n}=$ Sample Size

$\mathrm{Z}=\mathrm{Z}$-value $=1.96$

$\mathrm{P}=$ Percentage of population picking a choice $=0.12$

$\mathrm{d}=$ Confidence interval $=20 \%$ of $\mathrm{P}=0.024$

$\alpha=0.05$

Therefore, the sample size (n) was 705 participants

\subsubsection{Sampling Procedures}

Considering the high number of sample size, the total survey was done in the area.

After the participants agreed to sign a consent form to participate this study, a face-to-face interview using electronic questionnaire was performed. The questionnaire was checked for accuracy (IOC) by three pharmacologists to increase survey reliability. The questionnaire included three parts, i.e., 1) demographic and medical conditions including sex, age, educational level, occupation, income, medical payment, alcohol and smoking status, underlying diseases, pain symptoms and treatments, 2) NSAID use related behavior use including type of NSAIDs, dose, frequency, time and purpose and 3) NSAID sources. To identify the NSAIDs used, we showed an example of NSAIDs which found in this area and supplied by health centers, grocery stores, and pharmacies to the participants to identify whether they took these drugs before including ibuprofen, diclofenac, aspirin, mefenamic acid, piroxicam and specific COX-2 inhibitors.

Data were analyzed using statistical analysis software packages for computer (SPSS for Windows, version 23). Descriptive statistics included frequency, percentage, average, standard deviation and mode to analyze general data. Chi-square test was used to analyze the differences in the regular use of NSAID group by demographics, i.e., sex, age, education level, occupation and income. Risk factors related to the regular use of NSAIDs were determined by univariate and multivariate analysis.

\subsection{Qualitative Study}

The qualitative study was performed by group interviews to assess 1) knowledge and understanding of NSAID use, 2) knowledge and understanding of the factors associated with NSAID use, 3) reasons for choosing not to act or behavior conducted at risk of NSAID use and 4) problems of NSAID use from the quantitative study. Participants were purposively selected and categorized according to their living community. Each group comprised both regular and nonregular NSAID users. In-depth interviews were performed among the providers of NSAIDs in the community including medical personnel and shop owners who distributed NSAIDs. The participants' profiles were created based on information from questionnaires. The conversations during the interviews were recorded using a voice recorder. The data was collected continuously until no new information was added, i.e., data saturation was reached.

The recorded conversations were completely transcribed to text. Text-based data were manually sorted and coded. Data were revised, organized and summarized for analysis. The methods used included content analysis, direct quotations and selected words to give consideration to actual local words used by the participants.

\subsection{Ethical Considerations}

The study protocol, questionnaires and consent form were reviewed and approved by the Ethics Committee of the Royal Thai Army Medical Department. Written informed consent was obtained from all enrolled participants.

\subsection{Operational Definitions and Abbreviations}

Regular used $=$ taking aspirin or NSAIDs at least three times weekly more than three months in one year

NSAIDs $=$ nonsteroidal anti-inflammatory drugs

THB = Thai bath, which equal to approximately 0.03 USD

\section{Results}

\subsection{Quantitative Study}

Characteristics of the population in the study of 771 participants are listed in Table 1 . Approximately $60 \%$ of the participants were female and more than $80 \%$ were aged above 40 . Most participants had education level at primary and lower. Approximately 40\% were agriculturalists. One half of them had income less than 5,000 THB monthly. 
Table 1. Characteristics of the participants and prevalence of regular NSAID use

\begin{tabular}{|c|c|c|c|}
\hline Characteristics & Total $(\%)$ & No. of regular NSAIDs use (\%) & $p$-value \\
\hline \multicolumn{4}{|l|}{ Sex } \\
\hline Male & $282(36.6)$ & $15(5.3)$ & 0.095 \\
\hline Female & $489(63.4)$ & $42(8.6)$ & \\
\hline Age & $52.3 \pm 14.2$ & $54.5 \pm 14.8$ & 0.267 \\
\hline \multicolumn{4}{|l|}{ Education level } \\
\hline No education & $56(7.3)$ & $7(12.5)$ & 0.314 \\
\hline Primary school & $581(75.3)$ & $41(7.1)$ & \\
\hline Above primary school & $134(17.4)$ & $9(6.7)$ & \\
\hline \multicolumn{4}{|l|}{ Occupation } \\
\hline Non-occupation & $285(37.0)$ & $21(7.4)$ & \\
\hline Agricultural & $294(38.1)$ & $22(7.5)$ & \\
\hline Others & $192(24.9)$ & $14(7.3)$ & 0.997 \\
\hline \multicolumn{4}{|c|}{ Average income (THB/month) } \\
\hline$<5,000$ & $394(51.1)$ & $36(9.1)$ & \\
\hline$\geq 5,000$ & $377(48.9)$ & $21(5.6)$ & 0.058 \\
\hline \multicolumn{4}{|l|}{ Smoking Status } \\
\hline Nonsmoking & $555(72.0)$ & $43(7.7)$ & 0.732 \\
\hline Former Smoker & $93(12.1)$ & $7(7.5)$ & \\
\hline Current Smoker & $123(15.9)$ & $7(5.7)$ & \\
\hline \multicolumn{4}{|l|}{ Drinking Status } \\
\hline Nondrinking & $449(58.2)$ & $37(8.2)$ & 0.549 \\
\hline Former drinker & $106(13.8)$ & $6(5.7)$ & \\
\hline Current drinker & $216(28.0)$ & $14(6.5)$ & \\
\hline \multicolumn{4}{|l|}{ Diabetes mellitus } \\
\hline No & $647(84.4)$ & $50(7.7)$ & 0.467 \\
\hline Yes & $120(15.6)$ & $7(5.8)$ & \\
\hline \multicolumn{4}{|l|}{ Hypertension } \\
\hline No & $574(74.8)$ & $39(6.8)$ & 0.246 \\
\hline Yes & $193(25.2)$ & $18(9.3)$ & \\
\hline \multicolumn{4}{|l|}{ Dyslipidemia } \\
\hline No & $634(82.7)$ & $44(6.9)$ & 0.257 \\
\hline Yes & $133(17.3)$ & $13(9.8)$ & \\
\hline \multicolumn{4}{|l|}{ Chronic kidney diseases } \\
\hline No & $748(97.5)$ & $53(7.1)$ & 0.046 \\
\hline Yes & $19(2.5)$ & $4(21.1)$ & \\
\hline \multicolumn{4}{|l|}{ Gout } \\
\hline No & $743(96.9)$ & $53(7.1)$ & 0.095 \\
\hline Yes & $24(3.1)$ & $4(16.7)$ & \\
\hline \multicolumn{4}{|l|}{ Osteoarthritis } \\
\hline No & $687(89.6)$ & $47(6.8)$ & 0.068 \\
\hline Yes & $80(10.4)$ & $10(12.5)$ & \\
\hline
\end{tabular}


Table 1 shows that 63.4 percent were female, mostly population were aged above 40 , had educated in primary and lower 82.7 percent. 38.5 percent were agriculturalists. 51.1 percent had income less than 5,000 THB per month, and shows the relationship of one variable. Chronic kidney disease was associated with the regular used of NSAIDs with statistical significance. Other diseases were not associated with the use of NSAIDs in regular use, significant statistically.

Fifty-seven participants (7.4\%) took NSAIDs at least three times weekly for more than three months, indicating the prevalence of regularly used NSAIDs. In addition, $183(23.7 \%)$ had been using NSAIDs in the last three months but did not meet the criteria for regular use. Both groups obtained NSAIDs from general/health promoting hospitals $(62.3 \%)$, pharmacies $(39.7 \%)$ and grocery stores $(20.9 \%)$, and they may be received form multiple sources. Ibuprofen (58.3\%), diclofenac (14.7\%), mefenamic acid (12.7\%), aspirin (10.0\%), piroxicam (3\%), specific COX-2 inhibitors $(1 \%)$ and naproxen $(0.3 \%)$ were identified as the NSAIDs used in this community. In addition, $2.6 \%$ of participants used their relatives' NSAIDs.

Univariate analysis for the factors associated with the regular use of NSAIDs is shown in Table 3. Those who had chronic kidney disease were significantly associated with regular NSAID use. Table 2 demonstrates that pain at the right elbow and right wrist and hips or thighs was significantly associated with regular NSAID use. The participants were asked to rate the intensity of pain that required NSAIDs using pain scores. Regular users scored their pain at 7.1 \pm 2.1 , significantly higher than nonregular users (5.8 \pm 2.3$)$ (Independent $t$ test, $\mathrm{p}<0.001$ ).

Univariate and multivariate analysis showed that for those aged $\geq 60$, hip or thigh pain and pain scores were independently associated with regular NSAID use (Table 3).

Table 2. Prevalence of regular NSAID use among the participants with different symptoms and locations of pain

\begin{tabular}{llll}
\hline Characteristics & No. (\%) & No. of regular NSAIDs use (\%) & $p$-value \\
\hline Symptoms (N= 634) & & $19(9.3)$ & 0.640 \\
Headache & $205(32.3)$ & $8(10.7)$ & 0.478 \\
Toothache & $75(11.8)$ & $4(13.3)$ & 0.312 \\
Dysmenorrhea & $30(4.7)$ & $48(8.3)$ & 0.620 \\
Myalgia & $576(90.9)$ & & \\
\hline Locations of pain $(\mathbf{N}=\mathbf{5 7 7})$ & & $4(17.4)$ & 0.122 \\
Left shoulder & $23(4.0)$ & $4(10.8)$ & 0.543 \\
Left elbow & $37(6.4)$ & $5(11.9)$ & 0.388 \\
Left wrist & $42(7.3)$ & $22(8.7)$ & 0.897 \\
Left knee & $254(44.0)$ & $6(12.0)$ & 0.421 \\
Left ankle & $50(8.7)$ & $4(17.4)$ & 0.122 \\
Right shoulder & $23(4.0)$ & $6(20.0)$ & 0.034 \\
Right elbow & $30(5.2)$ & $7(18.4)$ & 0.033 \\
Right wrist & $38(6.6)$ & $25(10.2)$ & 0.205 \\
Right knee & $245(42.5)$ & $4(9.5)$ & 0.773 \\
Right ankle & $42(7.3)$ & $10(14.5)$ & 0.057 \\
Neck & $69(12.0)$ & $16(11.1)$ & 0.193 \\
Upper/middle back & $144(25.0)$ & $14(10.5)$ & 0.337 \\
Lower back & $133(23.1)$ & $20(13.4)$ & 0.016 \\
Hip/thigh & $149(25.8)$ & & \\
\hline
\end{tabular}

Table 2 shows the relationship of variables demonstrated that pain right elbow and right wrist, and hips/thighs were associated in regular use of NSAIDs, significant statistically. 
Table 3. Univariate and multivariate analysis for the factors associated with regular NSAID use

\begin{tabular}{|c|c|c|c|c|c|c|}
\hline & \multicolumn{2}{|l|}{ NSAIDs use } & \multirow{2}{*}{ Crude OR $(95 \% \mathrm{CI})$} & \multirow{2}{*}{$p$-value } & \multirow{2}{*}{$\begin{array}{l}\text { Adjusted } \\
\text { OR }(95 \% \mathrm{CI})\end{array}$} & \multirow{2}{*}{$p$-value } \\
\hline & non-regular (\%) & regular (\%) & & & & \\
\hline \multirow[t]{2}{*}{ Pain score } & & & 1.28 & $<0.001$ & 1.2 & 0.045 \\
\hline & & & $(1.1-1.5)$ & & $(1.0-1.5)$ & \\
\hline
\end{tabular}

\section{Age (years)}

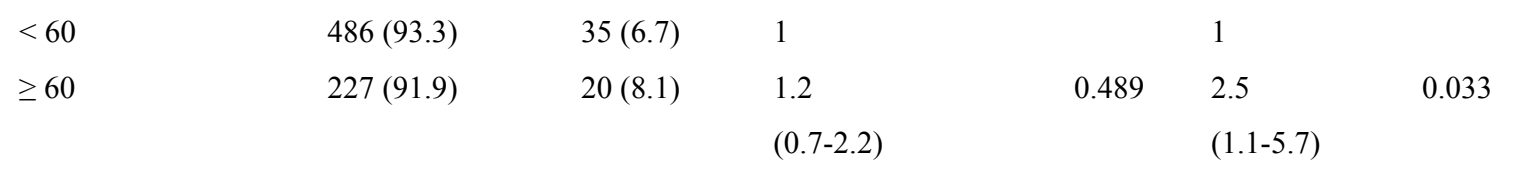

Pain at hip/thigh

\begin{tabular}{|c|c|c|c|c|c|c|}
\hline No & 399 (93.2) & $29(6.8)$ & 1 & & 1 & \\
\hline Yes & $129(86.6)$ & $20(13.4)$ & 2.1 & 0.012 & 3.0 & 0.008 \\
\hline & & & $(1.2-3.9)$ & & $(1.3-6.9)$ & \\
\hline
\end{tabular}

Pain at back

$\begin{array}{llllll}\text { No } & 400(92.4) & 33(7.6) & 1 & 1 & \\ \text { Yes } & 128(88.9) & 16(11.1) & 1.5 & 0.196 & 2.1 \\ & & (0.8-2.8) & (0.9-5.0) & 0.093\end{array}$

\section{Source of drugs}

$\begin{array}{lllllll}\text { Others } & 74(82.2) & 16(17.8) & 1 & & 1 & \\ \text { Hospital } & 108(72.5) & 41(27.5) & 1.8 & 0.089 & 1.7 & 0.235 \\ & & & (0.9-3.4) & & (0.7-4.2) & \end{array}$

\section{Chronic kidney diseases}

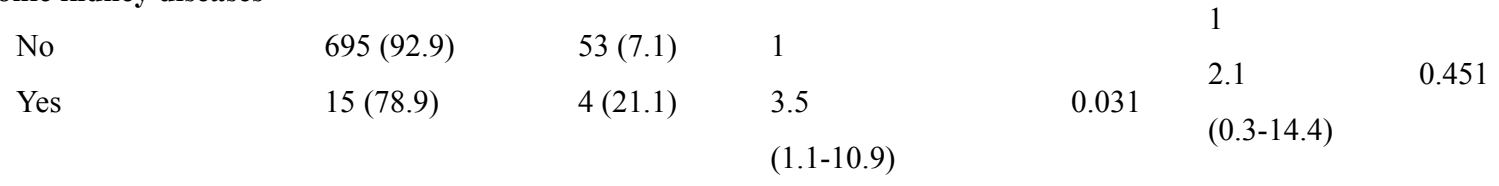

\section{Osteoarthritis}

\begin{tabular}{|c|c|c|c|c|c|c|}
\hline No & $640(93.2)$ & $47(6.8)$ & 1 & & 1 & \\
\hline \multirow[t]{2}{*}{ Yes } & $70(87.5)$ & $10(12.5)$ & 1.9 & 0.072 & 1.1 & 0.836 \\
\hline & & & $(0.9-4.0)$ & & $(0.4-3.3)$ & \\
\hline
\end{tabular}

Table 3 shows the relationship of multivariate analysis using multiple logistic regression analysis. Pain score, age, hip/thigh and the pain were associated with the use of NSAIDs in regular use, significant statistically. Adjusted for sex, income, hypertension, pain at left shoulder, right shoulder, right elbow, right wrist, neck, and upper/middle back

\subsection{Qualitative Study}

The results of qualitative research based on interviews with specific groups (group interview) and individual 
in-depth interviews (in-depth interview). Group interviews included both regular used NSAIDs and the drugs user but non-regular used in the community. 5 cases for in-depth interview also included drugs supplier, hospital staffs, pharmacist, and a grocery-store owner. The detailed results of the research are as follows.

\subsubsection{Characteristics of the Population}

Two group interviews comprised those who attend the entire group of 10 participants, there were seven women, average age of participants was 45 years old most of the participants is agriculture. The educational level of the participants was primary school. The average income of the participants was less than 5000 THB.

\begin{tabular}{lll}
\hline Group interview & Group 1 & Group 2 \\
\hline Participants & 4 & 6 \\
Sex & 4 females & 3 males, 3 females \\
Average age & 39.75 years old & 48.60 years old \\
Occupation & agriculture & agriculture, trader \\
Education & primary school & primary school \\
Average income (THB) & less than 5,000 & less than 5,000 \\
\hline
\end{tabular}

In-depth interviews consisted of five men, one woman, average age of the participants were 40.6 years old. The average income of the participants were 10,000-15,000 THB.

\begin{tabular}{lllll}
\hline In-depth interview & Non-regular user & Hospital staff & Pharmacists & Shop owners \\
\hline number & 1 person & 1 person & 1 person & 2 persons \\
sex & female & female & male & all 2 female \\
Average age (year) & 51 & 28 & 50 & 33.5 \\
Occupation & daily contractor & nursing assistant & pharmacist & personal business \\
Education & primary school & bachelor & bachelor & primary school \\
Average revenue (THB) & Less than 5,000 & $10,000-15,000$ & more than 20,000 & $15,000-20,000$ \\
\hline
\end{tabular}

\subsection{The Villagers Used NSAIDs because of Their Effectiveness}

Participants, who used NSAIDs, thought it could effectively and immediately reduce pain. The efficacy of NSAIDs was higher than common painkillers such as paracetamol. They considered that NSAIDs were medications for severe pain as mentioned by a 43 -year-old male farmer: "The pink tablet (ibuprofen) can dramatically improve my pain symptom. I was energized suddenly, without pain anymore" and a 28 -year-old female registered nurse also reported: "When the doctor once prescribed this medication and it works, they'll remember the pill and request the same one."

Pain was usually related to their work, mostly agriculture. The common reason for using NSAIDs was that they could work without pain troubles after taking these pills. A 32-year-old female entrepreneur said: "I may work too much, but when I took it, I could continue my work".

\subsection{Sources of NSAIDs in the Community}

Most participants thought accessing health services in their community was not too difficult. However, they were not satisfied and these health services did not meet their needs. This might be due to long waiting time, failure to treat pain symptoms and that the most prescribed analgesic drug was paracetamol. When they wanted stronger painkillers (referring to NSAIDs), they had to go to the hospital many times because they received small quantities. Thus, they will go to a grocery store to buy NSAIDs illegally. An 80-year-old male freelancer commented: "It's easier to buy the pills ourselves because it takes too long to see a doctor and sometimes we got only paracetamol". This information was validated by a 28 -year-old female registered nurse who confirmed: "We mainly prescribe paracetamol as the first line drug for pain. After that, we will consider giving NSAIDs when they still have the pain because NSAIDs have many side effects and are more dangerous". 
Alternatively, the villagers could buy NSAIDs with no prescription from a pharmacy or grocery store in the community. NSAIDs were requested according to the patients' need, not the indications. A 50-year-old male pharmacist affirmed: "I won't sell those (NSAIDs) to everyone because of side effects, but consumers remember that they must have been told that when they had severe pain then they would get it." A 32-year-old female merchant stated: "Most (consumers) wouldn't tell their symptoms, they just asked for the drug they wanted. If I had, I sold."

\subsection{Benefits of NSAIDs and Knowledge about Side Effects}

Most participants were aware of the side effects of NSAIDs including the main side effects on the gastrointestinal tract such as irritating stomach, stomachache and GI hemorrhage. A 55-years-old female daily contractor revealed: "My mother took this drug too much, and she got gastric bleeding then she died. The healthcare worker told that it affects our stomach, liver and kidney."

However, they still used them because pain-relieving effects made them better able to work. When they still presented pain symptoms without medication they could not work effectively, especially during the harvest season when they needed to harvest crops over a long time. Although the side effects of NSAIDs were explained, the villagers considered working was more important. A 48-year-old man farmer declared: "I'm scare about side effects, but I have to take it in order to continue my work". The administration and side effects of NSAIDs were better explained by health care professionals such as pharmacist. A 50-year-old man pharmacist revealed: "I always tell them they shouldn't take it before meals and don't take too much because it can cause abdominal pain". However, the advisory was not provided to every consumer. A 32-year-old female merchant expressed: "I wouldn't advise them because I don't know about side effects, I told them to ask at the clinic"

\section{Discussion}

The present study determined the situation of NSAID use in a rural community of central Thailand. This community mainly comprised farmers and villagers aged more than 50 years, which is relatively similar to most rural communities in Thailand. Higher risk of musculoskeletal injuries and chronic pain may occur due to their physical activities and age-related conditions. Thus, NSAIDs are sometimes required for their conditions. In the present study, a total of $31.1 \%$ of the participants had been using NSAIDs in the last three months. Our survey revealed that the regular NSAID users in this community totaled $7.4 \%$ which was relatively similar to results from a large-scale survey in the US (Zhou et al., 2013). The 2005 and 2010 National Health Interview Survey (NHIS) showed the prevalence of regular use of aspirin and NSAIDs among U.S. adults aged 18 years and older was $19.0 \%$ and $12.1 \%$, respectively (Zhou et al., 2013). However, source of NSAIDs in this Thai rural community varied; not all participants got the drugs through the hospital prescription (Jarernsiripornkul, Phueanpinit, Pongwecharak, \& Krska, 2016). As described in a study by Sri-Ngernyuang (1996-1997), drugs including NSAIDs were available at various sources in rural communities including grocery stores indicating the failure of the drug regulation system. In addition, our qualitative information indicated that other factors such as unsatisfactory treatment results by health promoting hospitals and inconvenience of going to the hospital led villagers to obtain NSAIDs from other sources such as pharmacies and grocery stores. A recent study in Thailand indicated that the adverse effects of NSAIDs were less disseminated compared with drug administration and indication in the university hospital setting (Jarernsiripornkul et al., 2016). Approximately $20 \%$ of the participants bought NSAIDs from grocery stores in the areas. These people may not have received proper information of NSAIDs including drug administration, indication and adverse effects. From the qualitative study, participants knew that NSAIDs could cause gastrointestinal adverse effects. However, the commonly used NSAIDs in this community including ibuprofen, diclofenac, mefenamic acid and aspirin also caused other adverse effects, some of which might be less common but severe including renal failure. NSAIDs affect renal functions including water and sodium retention, decreased renal blood flow, electrolyte imbalances and acute and chronic renal failure (Winkelmayer, Waikar, Mogun, \& Solomon, 2008; Radford et al., 1996; Abraham \& Keane, 1984). NSAIDs should be avoided (except aspirin) for patients with chronic kidney disease (CKD) because NSAIDs have been associated with disease progression among individuals with CKD (Winkelmayer, 2008; Radford et al., 1996; Abraham \& Keane, 1984). In the present study, approximately $20 \%$ of (4 of 19) patients with CKD regularly used NSAIDs. Some participants especially the elderly in the community were likely to have multiple comorbid conditions such as hypertension and diabetes mellitus. Regular use of NSAIDs may interact with commonly prescribed medications possibly altering drug effectiveness or increased risk of adverse effects. For example, NSAIDs can induce increased blood pressure and may potentially reduce the efficacy of commonly used antihypertensive agents including diuretics, beta-blockers and ACE inhibitors (Polónia, 1997).

Univariate and multivariate analysis showed that participants aged more than 60 years were significantly 
associated with regular NSAID use in this population. Risk of gastrointestinal, cardiovascular and renal adverse effects increased among elderly NSAIDs users. Thus, NSAIDs should be rarely prescribed and used with extreme caution among the elderly. In addition to the renal adverse effects mentioned above, the risk of gastrointestinal bleeding with NSAIDs increases with age. NSAID cardiovascular effects including fluid retention, hypertension, congestive heart failure, myocardial infarction and cerebrovascular accidents increase significant risk to the older age group as well. From the qualitative study, the adverse effects of NSAIDs mentioned by the participants were mainly gastrointestinal including stomachache and bleeding. Mortality caused by NSAID use in this community was mentioned by one of the participants.

Although NSAID adverse effects were emphasized by health care workers and pharmacists in this population, the villagers considered that these drugs could make them continue working. Common clinical problems in which NSAID use may be needed include acute or chronic pain from musculoskeletal injuries or degenerative conditions such as osteoarthritis which interrupted their working activities. We asked the participants to rate the highest pain scores to trigger their NSAID use. Our result showed that regular NSAID users had significantly higher pain score compared with the nonregular users. Thus, the patients with higher pain level needed more NSAIDs to relieve their pain regularly. Multivariate analysis also identified the pain score as an independent factor associated with regular NSAID use. Univariate analysis showed that pain at the right wrist, right elbow and hips or thighs were associated with regular NSAID use; however, multivariate analysis identified only pain at the hips or thighs was an associated independent factor. Joint and muscle pain was related to their occupation; most were farmers. Because they were concerned about not being able to work during farming season, they had to maintain their working ability using potent drugs like NSAIDs. Our qualitative data showed that most participants knew of the side effects of NSAIDs. However, they still used them for financial reasons. Safe pain management must be considered in the rural community to avoid the negative impact of regular NSAID use which may include a proper drug administration such as low dose/short term use in the high risk group. In addition, nonpharmacologic approaches, such as physiotherapy, exercise and local therapies, may be useful.

This information could be used to plan public health policy specific to legitimate pharmaceutical distribution. The Thai Food and Drug Administration should monitor the distribution of illegal drugs and campaign to execute selling and purchasing of correct doses. Side effects of pain medications should be well disseminated to the public including how to use pain medications as required. Most people know about the side effects, so not only could behavior modification be implemented, educating the public regarding proper alternative treatments may reduce the rate of drug use. However, this study was conducted in a limited population. Further studies should be conducted in all different regions of Thailand to determine the situation of regular NSAID use at the national level.

In conclusion, the prevalence of regular NSAID use in this rural community was $7.1 \%$. Univariate and multivariate analysis showed that higher pain scores, age of more than 60 years and pain at the hips or thighs were associated with regular NSAID use. Most people with joint and muscle pain from heavy use were related to their occupation. They took NSAIDs to reduce the pain to be able to continue working. Another result was despite the public health system that aims to serve Thais properly; many patients had unsatisfactory treatment results, so some villagers chose to buy the drugs themselves from pharmacies or grocery stores. Most participants had understanding side effects of NSAIDs. However, they still used them because the pain-relieving effects could allow them to work in the following days to meet their economic needs at low financial status.

\section{Acknowledgments}

We gratefully acknowledge support, including funds and operation, from the Department of Military and Community Medicine, Department of Pharmacology, especially Professor Major Colonel Mathirut Mungthin for revising and advising, and medical students, Pajjekpol Juansang, Charinee Kantasiripitak, Attaya Raksuan, Santiphap Wiwekwin, Sirada Suethiensakul, Boriboon Wangkaew, Nissa Jongudomsombat, Tanadon Treechairasmee, Unchisa Kittisattra, Rachanont Sununtyeunyong, Issara Chandrsawang, Pawaris Wangkiat and Sakdinant Imsuwan, Phramongkutklao College of Medicine, local village health volunteer in Baan Na Yao Village and all participants for their help in this research.

\section{Competing Interests Statement}

The authors declare that there are no competing or potential conflicts of interest.

\section{References}

Abraham, P. A., \& Keane, W. F. (1984). Glomerular and interstitial disease induced by non-steroidal anti-inflammatory drugs. Am J Nephrol., 4(1), 1-6. https://doi.org/10.1159/000166764

Akepalakorn, W. (2009). The 4th Thai National Health Survey by Physical Examinations 2551-2 BE. Bangkok, 
National Health Examination Survey Office. 2009: 114-117. Retrieved from: http://www.hiso.or.th/hiso/picture/reportHealth/report/report1.pdf

Jarernsiripornkul, N., Phueanpinit, P., Pongwecharak, J., \& Krska, J. (2016). Experiences of and attitudes towards receiving information about non-steroidal anti-inflammatory drugs: A cross-sectional survey of patients in Thailand. Expert Opin Drug Saf., 15(4), 417-26. https://doi.org/10.1517/14740338.2016.1139571 PMid:26742855

Laine, L. (2001). Approaches to nonsteroidal anti-inflammatory drug use in the high-risk patient. Gastroenterology. 120(3), 594-606. https://doi.org/10.1053/gast.2001.21907 PMid:11179238

Lertsrisatit, P., \& Jantawiyanuchit, S. (2008). Non-steroidal Anti-inflammatory drugs. Faculty of Ramathibodi hospital. $2008 . \quad$ Retrieved from http://med.mahidol.ac.th/med/sites/default/files/public/pdf/medicinebook1/NSAIDS.pdf

Page, J., \& Henry, D. (2000). Consumption of NSAIDs and the Development of Congestive Heart Failure in Elderly Patients. Arch Intern Med., 160(6), 777-84. https://doi.org/10.1001/archinte.160.6.777 PMid:10737277

Polónia, J. (1997). Interaction of antihypertensive drugs with anti-inflammatory drugs. Cardiology. 88 Suppl 3:47-51. https://doi.org/10.1159/000177507

Pope, J. E., Anderson, J. J., \& Felson, D. T. (1993). A meta-analysis of the effects of non-steroidal anti-inflammatory drugs on blood pressure. Arch Intern Med, 153, 477-84. https://doi.org/10.1001/archinte.153.4.477 PMid:8435027

Radford, M. G., Jr., Holley, K. E., Grande, J. P., Larson, T. S., Wagoner, R. D., Donadio, J. V., et al. (1996). Reversible membranous nephropathy associated with the use of non-steroidal anti-inflammatory drugs. JAMA, 276(6), 466-9 https://doi.org/10.1001/jama.276.6.466 PMid:8691554

Somia, G., \& Maria, A. (2014). Prevalence of prescribing pattern of more than one NSAIDs in Pakistan. $J$ Sci Innovative Res, 3(Suppl 2), 148-54.

Sorensen, H. T., Mellemkjaer, L., Blot, W. J., et al. (2000). Risk of upper gastrointestinal bleeding associated with use of low-dose aspirin. Am $J$ Gastroenterol., $95(9), \quad$ 2218-24. https://doi.org/10.1111/j.1572-0241.2000.02248.x, https://doi.org/10.1016/S0002-9270(00)01040-6

Sri-Ngernyuang, L. (1997). Drug abundance: situation of drugs and drug distribution in the villages of rural Thailand. Asia Pac J Public Health, 9, 18-23. https://doi.org/10.1177/101053959700900104 PMid:10050194

Winkelmayer, W., Waikar, S., Mogun, H., \& Solomon, D. (2008). Nonselective and Cyclooxygenase-2-Selective NSAIDs and Acute Kidney Injury. Am J Med., 121(12), 1092-1098. https://doi.org/10.1016/j.amjmed.2008.06.035 PMid:19028206

Wolfe, M. M., Lichtenstein, D. R., \& Singh, G. (1999). Gastrointestinal toxicity of non-steroidal anti-inflammatory drugs. $N$ Engl J Med, 340(24), 1888-99. https://doi.org/10.1056/NEJM199906173402407 PMid:10369853

Zhou, Y., Boudreau, D., \& Freedman, A. (2013). Trends in the use of aspirin and nonsteroidal anti-inflammatory drugs in the general U.S. population. Pharmacoepidemiol Drug Saf., 23(1), 43-50. https://doi.org/10.1002/pds.3463 PMid:23723142

\section{Copyrights}

Copyright for this article is retained by the author(s), with first publication rights granted to the journal.

This is an open-access article distributed under the terms and conditions of the Creative Commons Attribution license (http://creativecommons.org/licenses/by/4.0/). 